\title{
Chapter
}

\section{HTTP Networking}

HTTP networking is ubiquitous on mobile devices. This book would certainly not be complete if it did not include a chapter explaining how to use the BlackBerry 10 networking services. In this chapter, I am going to exclusively concentrate on HTTP networking, which covers about 90 percent of the cases you will face during application development. Also, BlackBerry 10 leverages the underlying QtNetwork module, which makes HTTP programming amazingly simple. The goal of this chapter is to show you how the different networking classes work together to access HTTP servers from a BlackBerry 10 mobile device.

An immediate application of networking is obviously to build a "rich thin client" where you use Cascades to build your application's native user interface and remotely access business logic implemented as rest services. By now you must have realized that Cascades and QML make user interface design a snap. Adding networking to the mix just opens a completely new dimension of connected applications. For example, exposing enterprise services securely to your workforce-something that BlackBerry has always been at the forefront with-is an obvious practical application.

After having read this chapter, you will have a good understanding of

- The Qt networking classes.

- How to use the networking classes to build connected Cascades applications.

How to design responsive Uls by handling network requests and replies asynchronously.

Another important goal of this chapter is to illustrate all the concepts introduced so far by writing a slightly more complex app than the ones demonstrated so far. The application will take the form of a Cascades client app for a remote weather REST service and will emphasize the separation of UI logic from the core business logic written in $\mathrm{C}++$. The application will also show you how to breakdown your $\mathrm{C}++$ code in classes with delimited responsibilities. 


\section{Qt Networking Classes}

HTTP networking using Qt mostly involves the following classes:

ONetworkAccessManager: This class allows you to send network requests and receive replies. The ONNetworkAccessManager's API is entirely asynchronous, thus guaranteeing that the user interface thread is not blocked during an HTTP request.

- ONetworkRequest: This class encapsulates all the required information for an HTTP request. Typically, you will be using ONetworkRequest's url property to access an HTTP URL.

- ONetworkReply: This is ONetworkRequest's counterpart; it encapsulates the data received from the server.

\section{QNetworkAccessManager}

ONetworkAccessManager is the grand dispatcher of all the network interactions in your application. You will generally use a single instance of this class to handle all the networking logic of your app. The ONetworkAccessManager object holds the common configuration and settings for the requests it sends. It should be noted that all functions in this class are reentrant. This means that you can call the class methods multiple times, even if a given network request has not yet completed (this is also possible because the class methods are asynchronous, or in other words, nonblocking). If necessary, the QtNetworkAccessManager internally queues the requests it receives, but has the capability to process multiple requests concurrently. The following is a review of ONetworkAccessManager's most important methods:

ONetworkReply* ONetworkAccessManager: :get (const ONetworkRequest\& request): Posts a request to obtain the contents of the target specified by request. For the HTTP protocol, the request corresponds to the HTTP GET request. Returns a pointer to a ONetworkReply object, opened for reading, which can be used to retrieve data as soon as it is available.

ONetworkReply* ONetworkAccessManager: : post (const ONetworkRequest\& request, const QByteArray\& data): Sends an HTTP POST request to the destination specified by request and returns a pointer to a ONetworkReply object opened for reading. ONetworkReply contains the server's response.

The QByteArray instance contains the data to be uploaded to the server.

- ONetworkReply* ONetworkAccessManager: :post(const ONetworkRequest\& request, OIODevice* data): Similar to the previous method, but this time the posted data is passed as a pointer to a OIODevice object. In other words, you can use this method to post the contents of a file by passing a OFile object as the second method parameter (this is possible because OFile inherits from QIODevice).

- ONetworkReply* ONetworkAccessManager: :post(const ONetworkRequest\& request, OHttpMultipart* multipart): Posts the content of a multipart message to the destination identified by request. 
ONetworkReply* ONetworkAccessManager: :put(const ONetworkRequest\& request, const QByteArray\& data): Sends an HTTP PUT request to the destination specified by request and returns a pointer to a ONetworkReply object opened for reading. This method makes sense in the context of a REST service, where PUT is used for creating a resource and POST for updating or modifying one. The ONetworkReply object contains the optional server response. The QByteArray instance contains the data to be uploaded to the server. Just as with an HTTP POST request, the method is overloaded and can also take a OIODevice* and OHttpMultipart* as a second parameter.

- ONetworkConfiguration ONetworkAccessManager::configuration(): Returns the network configuration that will be used to create the network session.

void ONetworkAccessManager: :setConfiguration(const QNetworkConfiguration\& config): Sets the network configuration that will be used to create the network session.

- ONetworkCookieJar ONetworkAccessManager: :cookieJar(): Returns an instance of ONetworkCookieJar used to store cookies obtained from the network, as well as cookies about to be sent.

void ONetworkAccessManager: : setCookieJar(ONetworkCookieJar* cookieJar): Sets the manager's cookie jar. The cookie jar will be used by all requests dispatched by the network manager.

void ONetworkAccessManager: : setCache(OAbstractNetworkCache* cache): Sets the network manager's cache. The cache is used for all requests dispatched by the manager. You can use this function to specify an object that implements additional features, such as saving cookies to permanent storage or caching JavaScript and CSS files. Note that, by default, the network manager does not cache data. OAbstractNetworkCache provides the interface for cache implementation. As implied by its name, OAbstractNetworkCache is an abstract base class that cannot be instantiated. Instead, you can use a ONetworkDiskCache, which provides a concrete implementation. You can also control cache configuration with the ONetworkRequest request object (this will be explained in the next section).

Note As mentioned previously, you should always reuse the same QNetworkAccessManager instance. Note that you can conveniently access the default declarative engine's QNetworkAccessManager instance by using the OMLDocument: : defaultDeclarativeEngine()->networkAccessManager () method call (because OMLDocument: : defaultDeclarativeEngine() is a static method, you can always access the associated default declarative engine from anywhere in your code). 


\section{QNetworkRequest}

A ONetworkRequest object holds a URL to be requested by a ONetworkAccessManager. You can specify the target URL using one of the following methods:

ONetworkRequest: : ONetworkRequest(const OUrl\& url = QUrl()): Constructs a new network request with url as the URL to be requested.

ONetworkRequest: : setURL(const QUrl\& url): Sets the URL this network request is referring to.

You can also provide additional information to further customize the request (for example, by setting header values, request priorities, and cache configurations). In the specific case of caching, you can specify the cache behavior by setting a ONetworkRequest's CacheLoadControlAttribute attribute, as follows:

ONetworkRequest: : setAttribute(ONetworkRequest: :CacheLoadControlAttribute, const QVariant\& value): Sets the cache behavior. The following are the possible values:

ONetworkRequest: :AlwaysNetwork: Always load from the network and do not check if the cache has a valid entry.

QNetworkRequest: : PreferNetwork: This is the default behavior; load from the network if the cache entry is older.

- ONetworkRequest: :PreferCache: Load from the cache first; otherwise, load from the network. Note that you risk loading stale data in this case.

- ONetworkRequest: :AlwaysCache: Always try to load from the cache. In other words, this option corresponds to an offline mode. Note that you can use ONetworkRequest: : PreferCache for specific file types, such as CSS and JavaScript, where you are certain that they will not change during the application's lifetime.

Because you can specify the cache behavior on a per request basis, this can be very convenient if you have multiple requests of different kinds. However, for the biggest majority of network requests, you can simply set the target URL and pass the request to the ONetworkAccessManager.

\section{QNetworkReply}

QNetworkReply encapsulates the server's response and provides all the necessary functionality for retrieving the received data. The class inherits from OIODevice, which is the abstract base class for devices supporting reading and writing blocks of data. You will generally use the QByteArray QIODevice: :read(qint64 maxSize) and QByteArray QIODevice: :readAll() methods to retrieve the data. The former method reads, at most, maxSize bytes from the device. The latter reads all available data from the device. Both methods return the data as a QByteArray.

The following summarizes ONetworkReply's most important methods:

bool ONetworkReply: :isRunning() const: Returns true if the corresponding request is still being processed.

- OByteArray ONetworkReply: :read(qint64 maxSize): Inherited from OIODevice; see description given at the start of this section. 
- OQByteArray ONetworkReply: :readAll(): Inherited from OIODevice; see description given at the start of this section.

- ONetworkRequest ONetworkReply: :request(): Returns the request that was posted for this reply.

- QUrl ONetworkReply: :url(): Returns the URL of the content downloaded or uploaded. Note that the URL may be different from the one specified in the original request.

- NetworkError ONetworkReply: : $\operatorname{error}():$ Returns the error that was found during the processing of this request. Returns ONetworkReply: :NoError if the request was processed successfully. Check the API documentation for all the possible values taken by the ONetworkReply: :NetworkError enumeration.

- OVariant ONetworkReply: :attribute(Attribute code, const OVariant\& defaultValue $=$ QVariant ()$)$ : Returns the attribute associated with code. If code has not been set, returns defaultValue. Attributes are metadata that are used to pass additional information from the reply back to the application. As you will see in the examples section, you will use this property to detect HTTP redirects.

- ONetworkReply: :abort(): Aborts the operation immediately and closes any network connections still open.

ONetworkReply can also emit the following signals:

- ONetworkReply: : finished(): This signal is emitted when the reply has finished processing. The data can be retrieved by calls to ONetworkReply: :read() or ONetworkReply: :readAll().

- ONetworkReply::downloadProgress(qint64 bytesReceived, qint64 bytesTotal): This signal is emitted to indicate the data download's progress for a given network request. The download is finished when bytesReceived is equal to bytesTotal. Note that you should handle this signal when large amounts of data are being downloaded to convey some feedback to the user (for example, by displaying a Cascades ProgressIndicator). (You can also opt to process the data in chunks, as it becomes available.) The bytesReceived parameter indicates the number of bytes received, whereas bytesTotal indicates the total number of bytes expected to be downloaded. Note that if the total number of bytes to be downloaded is unknown, bytesTotal will be -1 , but when the download has completed bytesReceived will always be equal to bytesTotal.

- ONetworkReply: :uploadProgress(qint64 bytesSent, qint64 bytesTotal): This signal is emitted to indicate the upload progress of a network request. The upload is finished when bytesSent is equal to bytesTotal.

- ONetworkReply: : sslErrors(const QList<QSslError $\rangle$ \& errors): This signal is emitted if the SSL/TLS session encountered errors during the setup, including certificate verification errors. The list of errors is provided by the errors parameter. 
Note You should always warn the user if ssl errors occur and give him the option to cancel the request.

\section{HTTP Networking Examples}

The examples provided in this section illustrate typical usage scenarios of the networking classes.

\section{HTTP GET}

Let's start with a simple GET request to access a REST service. The data in the response will be returned in JSON format. To parse the object, you will have to use an instance of the Cascades JsonDataAccess class and handle the JSON structure in-memory. The Qt object constructed from JSON by the JsonDataAccess instance will always be a OVariant that either contains a OVariantList (if an array of JSON objects is returned by the service) or a QVariantMap (if a single object is returned). The mapping between JSON types and Qt types is summarized as follows:

- int: Mapped to a OVariant(Int64). To access the contained int use QVariant: : toInt().

uint: Mapped to a QVariant(Uint64). To access the contained uint use QVariant: : toUInt().

- real: Mapped to a OVariant(double). To access the contained real use QVariant: :toReal().

- string: Mapped to a QVariant (const char*). To access the contained string use QVariant: : toString().

- boolean: Mapped to a QVariant(bool). To access the contained boolean use QVariant: : toBool().

- array: Mapped to a OVariant(OVariantList). To access the contained array use Qvariant: :toList().

- object: mapped to a OVariant(OVariantMap). To access the contained object, use OVariant: : toMap().

The requested URL corresponds to the list of categories defined in my WordPress blog and is given at http://aludin.com?json=get_category_index. Listing 7-1 shows you an example of the returned JSON object.

\section{Listing 7-1. JSON Response}

\{

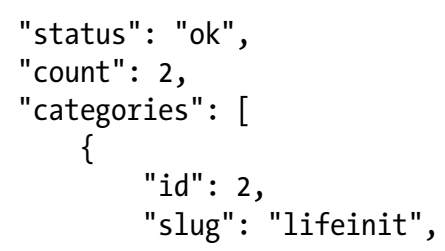




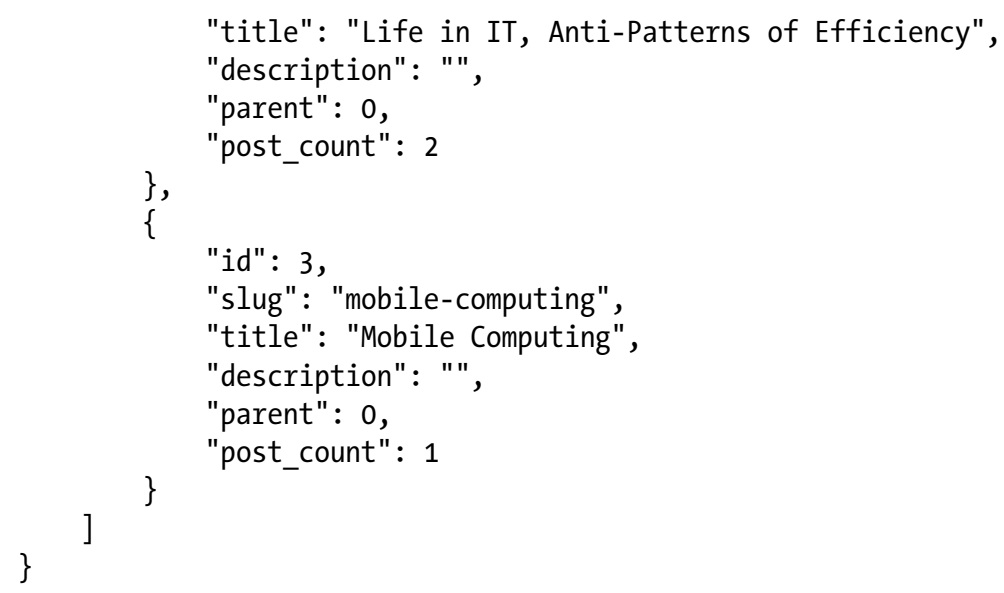

Listing 7-2 shows you how to perform the HTTP GET request to retrieve the JSON document displayed in Listing 7-1.

Listing 7-2. ApplicationUl::getCategories()

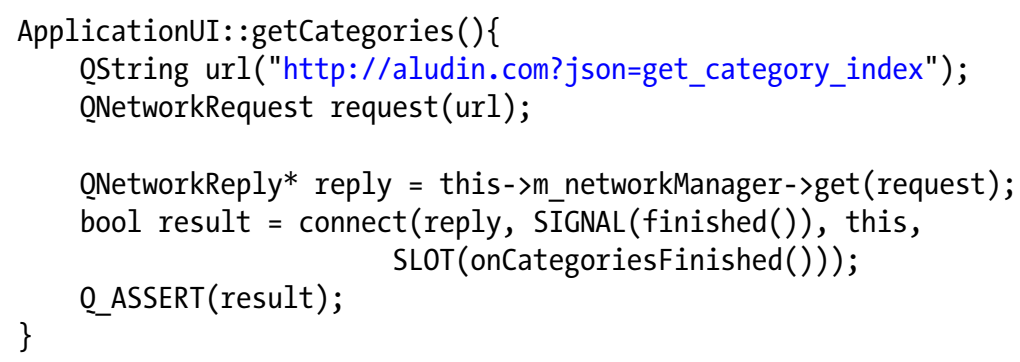

It is not shown in the previous code, but you can safely assume that ApplicationUI: :my_networkManager has been initialized with the default declarative engine's ONetworkAccessManager.

And Listing 7-3 illustrates how to perform the actual JSON response parsing once it has been returned by the service.

\section{Listing 7-3. ApplicationUl::onCategoriesFinished()}

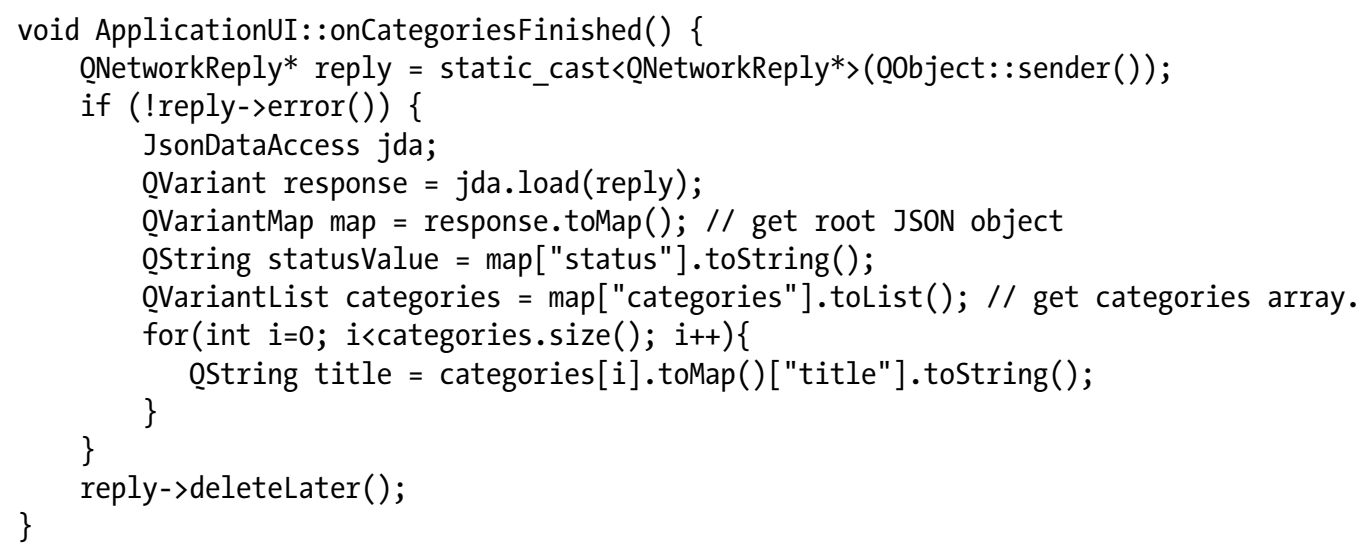


You will see later that you can conveniently chain the OVariant method calls to navigate the JSON object structure. Note that as a convenience and for clarity, I am using strings literals directly in the code, but ideally you should use string constants to avoid sprinkling your code with literals.

Finally, if your request takes additional parameters, you should use URL encoding to make sure that the parameters do not contain reserved HTTP characters (see Listing 7-4).

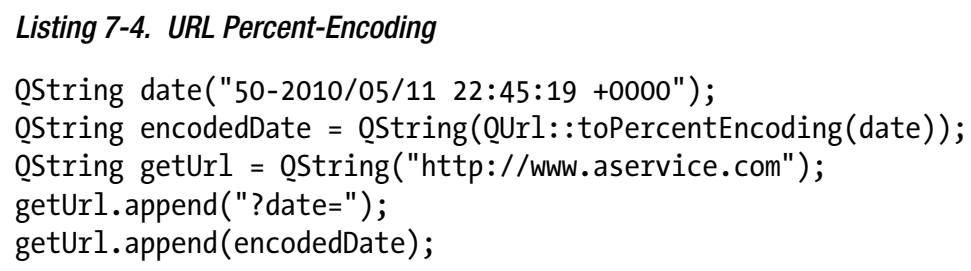

Listing 7-4. URL Percent-Encoding

OString date("50-2010/05/11 22:45:19+0000");

OString encodedDate = OString (QUrl:: toPercentEncoding (date));

ôString getUrl = OString("http://www. aservice.com");

getUrl. append("?date=");

getUrl.append(encodedDate);

\section{HTTP POST}

Posting data is just as simple as performing HTTP GET requests. You will have to specify the data parameters by adding them to a OByteArray. You also need to make sure that you separate each parameter-value pair with an ampersand, as shown in Listing 7-5.

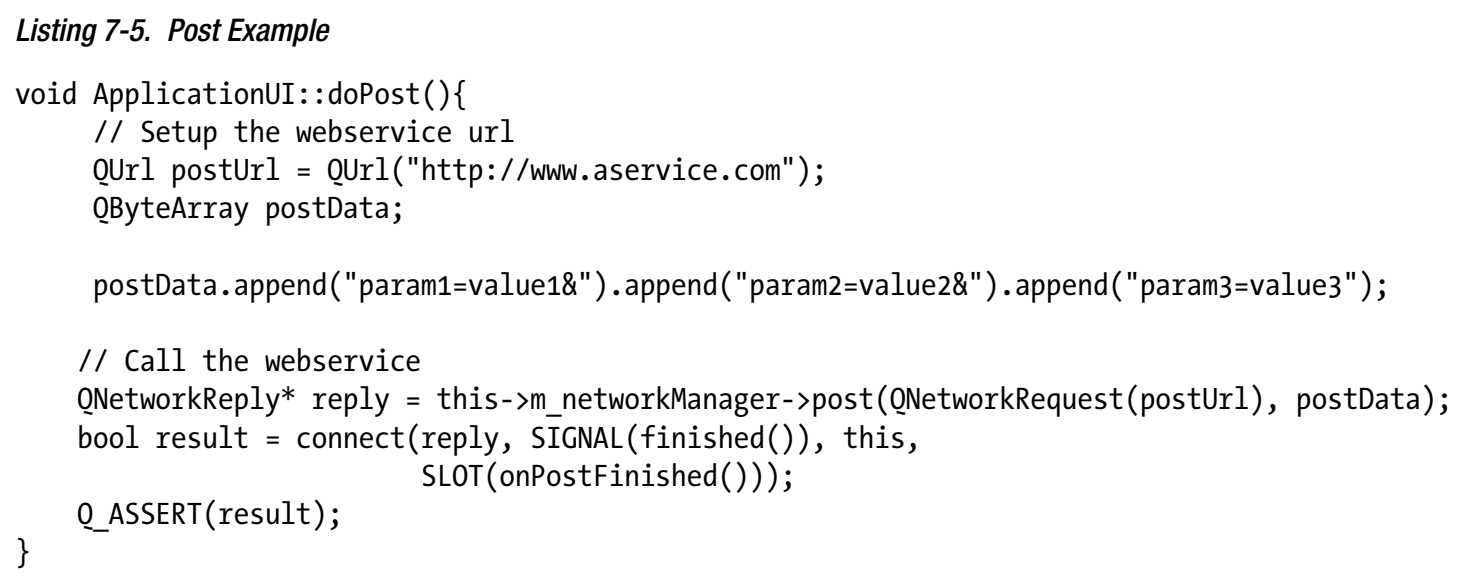

Once again, in practice you should use percent-encoding for the parameters you pass to the POST request. Also, in the onPostFinished() slot, don't forget to release the ONetworkReply instance using ONetworkReply: :deleteLater().

\section{Handling an HTTP Redirect}

At certain times, you will have to process an HTTP redirect. A redirect is not an error and simply indicates that a resource has moved. Listing 7-6 shows you how to handle the situation. 


\section{Listing 7-6. Redirect Check Example}

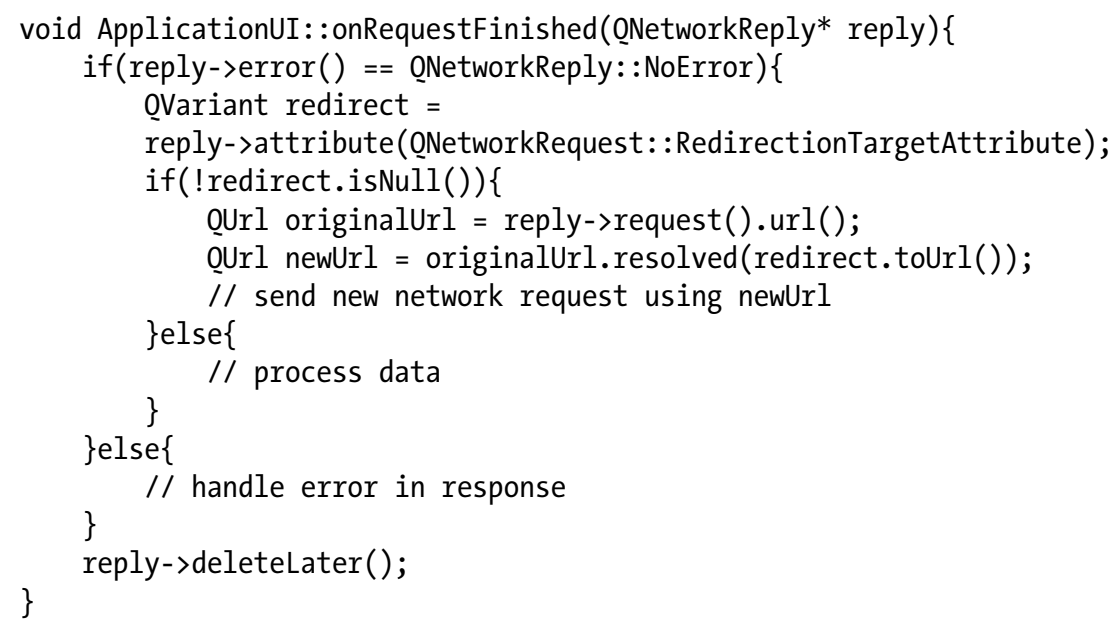

In practice, you should always be ready to handle HTTP redirects.

\section{Handling Authentication}

Certain HTTP services will require authentication before providing you access to their resources. In those cases, you can use the ONetworkAccessManager: : authenticationRequired(ONetworkReply* reply, OAuthenticator* authenticator) signal to handle the authentication request. Listings 7-7 and 7-8 illustrate how to implement authentication in your own code.

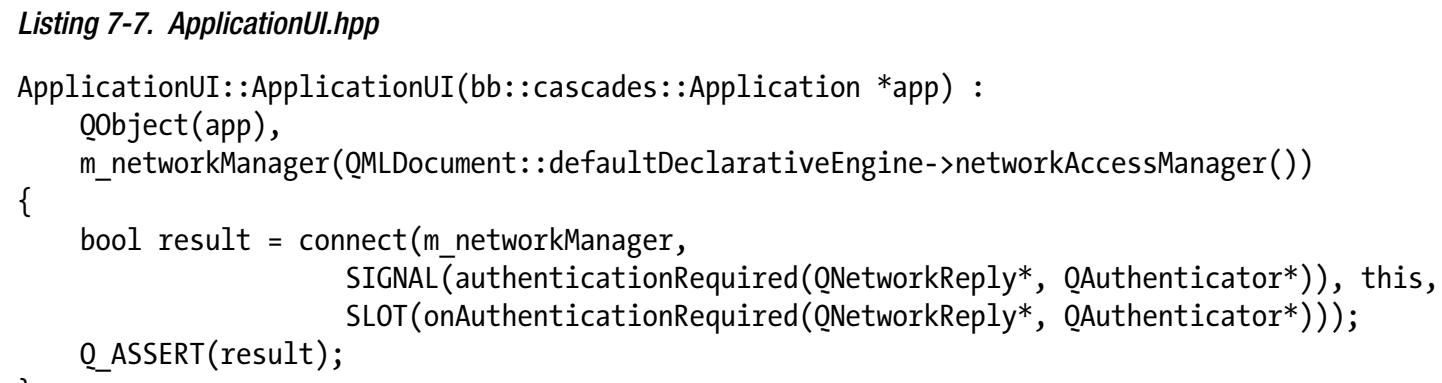

\section{Listing 7-8. ApplicationUl.cpp Authentication Handler}

void ApplicationUI: : onAuthenticationRequired(ONetworkReply* reply,

SystemCredentialsPrompt prompt $=$ new SystemCredentialsPrompt; prompt->exec();

authenticator->setUser (prompt->usernameEntry ()$)$;

authenticator->setPassword (prompt->passwordEntry ());

prompt->deleteLater(); 
The ONetworkAccessManager: : authenticationRequired(ONetworkReply*, QAuthenticator*) signal is connected to the corresponding slot in the application delegate's constructor. Therefore, whenever a server request needs to be authenticated, the slot will be called. As shown in Listing 7-8, you can use a SystemCredentialsPrompt object to display a modal dialog requesting the user's credentials (see Figure 7-1). Note that the majority of Cascades controls methods are nonblocking (in other words, they return immediately and processing continues). However, in this specific case, we want to be able to call a blocking method until the user has provided his credentials. To achieve this behavior, you should call SystemCredentialsPrompt: :exec() instead of SystemCredentialPrompt: : show(), which is the nonblocking version. (Internally, SystemCredentialsPrompt: : exec() creates a nested event loop to provide the blocking functionality. When the nested event loop is exited, control is returned to the main event loop). Note that once you have finished with the prompt object, you must call Oobject: : deleteLater() instead of deleting the object immediately.

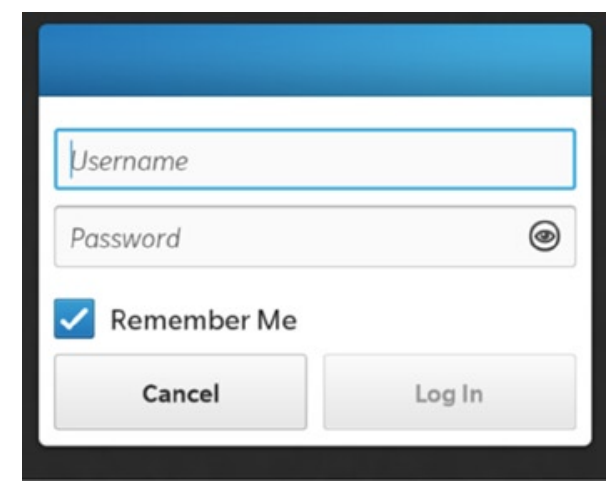

Figure 7-1. Credentials prompt

Finally, the authenticator should be updated with the user's credentials, which are sent back to the server.

\section{Weather2}

I promised you in Chapter 2 that we would build a weather app relying on the REST service introduced at the time. In essence, I want to illustrate how you can design an enticing Cascades UI on top of raw data (which would be the JSON document returned by the weather service). You will also learn how to combine multiple services together (such as Google Maps) to further enrich your application. Finally, you will see how the networking classes are used in practice to perform asynchronous requests. The application we are about to design is called, quite appropriately, Weather2 (the default Weather app is bundled with BlackBerry 10). The finished application's UI is shown in Figures 7-2, 7-3, and 7-4. The application has two tabs. On the first tab, you can perform a query by country, state, or city using a text field. If your query returns multiple results, the application will ask you to select a city from a list of values displayed in a SystemListDialog (see Figure 7-2). 


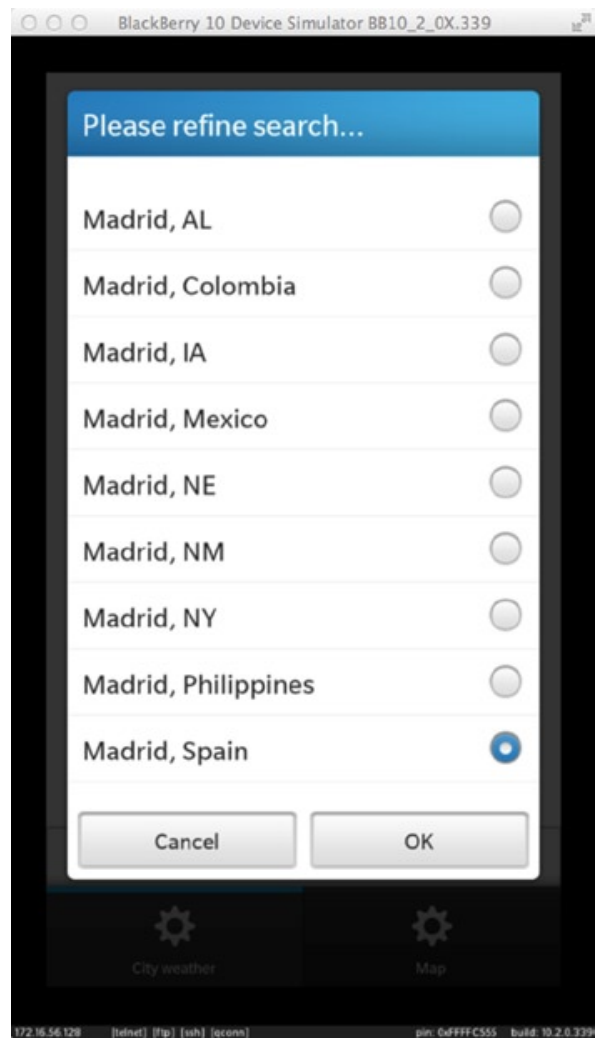

Figure 7-2. City selection

As soon as you have selected a city, the weather conditions are displayed, including the city's latitude and longitude (see Figure 7-3). 


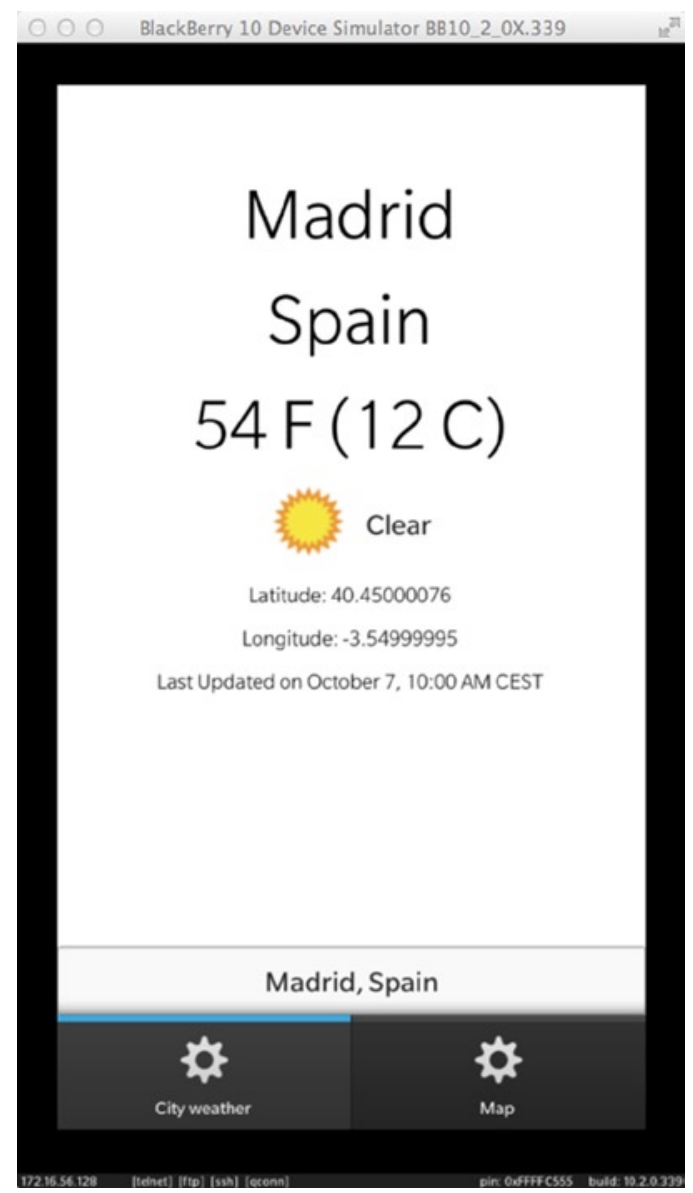

Figure 7-3. City view

If you select the second tab, a map will be displayed, with the city location highlighted by a small icon representing the weather conditions (see Figure 7-4). 


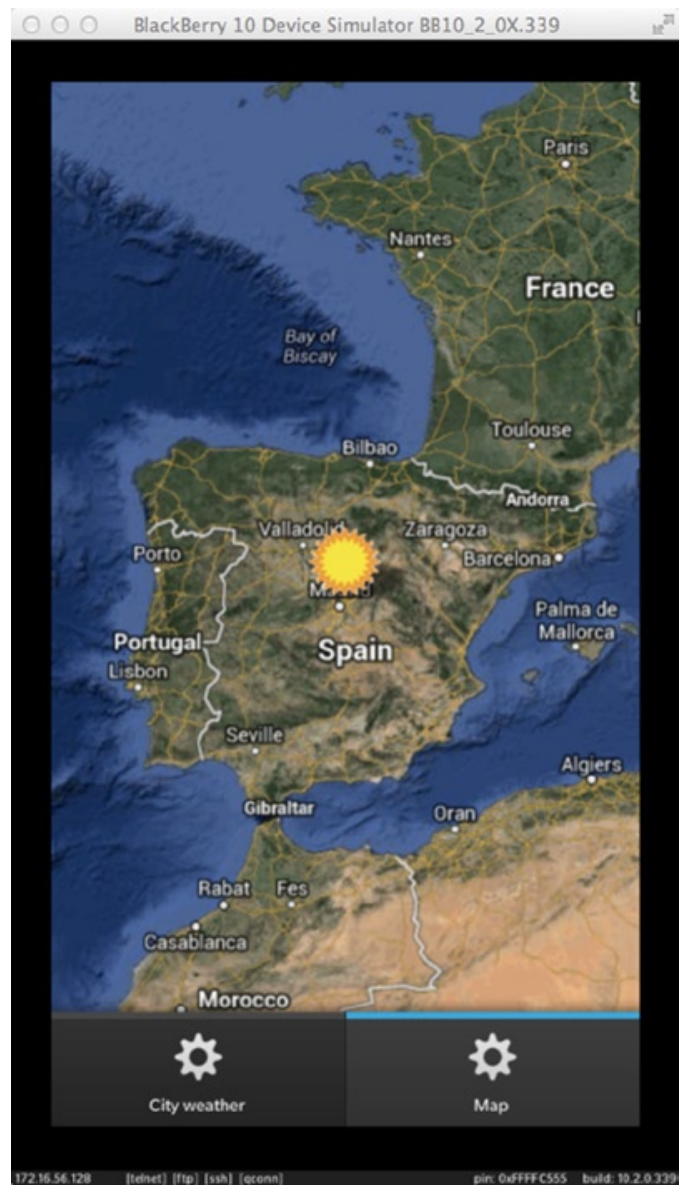

Figure 7-4. Map view

\section{Application Design}

Before actually looking at the app implementation, let's summarize once again the most important BlackBerry 10 design principles and recommendations (you can refer to Chapter 3 for a more detailed discussion of these points):

- Separate UI logic from business logic. Although it is possible to directly access Cascades controls from $\mathrm{C}++$, the preferable way to build BlackBerry 10 apps is by clearly decoupling the UI logic from the rest of the application's logic written in $\mathrm{C}++$. As stated in Chapter 3, one of the major strengths of QML and $\mathrm{C}++$ integration is the ability to implement the QML UI separately from $\mathrm{C}++$. The $\mathrm{C}++$ business logic can therefore be blissfully unaware of the QML layer (in other words, using Q0bject: : findChildren()to access Cascades controls by object name from $\mathrm{C}++$ is considered a bad practice because it adds tight coupling between UI and business logic). 
- Prefer signals for communicating between $\mathrm{QML}$ and $\mathrm{C}++$.

- Prefer properties and QML bindings to synch data between QML and C++ (you will also notice that at times I pass data as signal parameters). A QML component can have its properties bound to a C++ class' properties. If a $\mathrm{C}++$ property is updated, a signal has to be emitted from $\mathrm{C}++$ in order notify the QML declarative engine, which then updates the corresponding QML bound property. Note that bindings can be defined both ways: the declarative engine will also automatically update the $\mathrm{C}++$ bound property when the corresponding QML property changes.

- Break down your UI in multiple QML components instead of designing it as a single monolithic bloc. This will save you major headaches when you need to selectively update UI parts. Indeed, the ability to extend QML with your own custom components is a major advantage that you should leverage as much as possible.

Having emphasized these points, let's start with the UI design.

Note The source code for the Weather2 application can be found in this book's repository on GitHub at https://github.com/aludin/BB10Apress.

\section{Creating the UI}

Weather2's UI is split between four QML components:

main.qml: The QML document initially loaded by the application delegate. It defines a tabbed pane containing two tabs (see Listing 7-9).

- WeatherDetails.qml: The control responsible for handling user input for weather requests. The control also manages various system prompts for notifying or requesting additional information from the user, when necessary (you will see that the prompts are defined as attached objects).

- City.qml: The control responsible for displaying the weather data for a given city. Note that this control is referenced in WeatherDetails.qml (see Listing 7-10).

- WeatherMap.qml: The control responsible for displaying a map with the weather conditions for the selected city (see Listing 7-11).

\section{Listing 7-9. main.qmI}

import bb.cascades 1.2

TabbedPane \{

id: tabbedPane

showTabsOnActionBar: true 


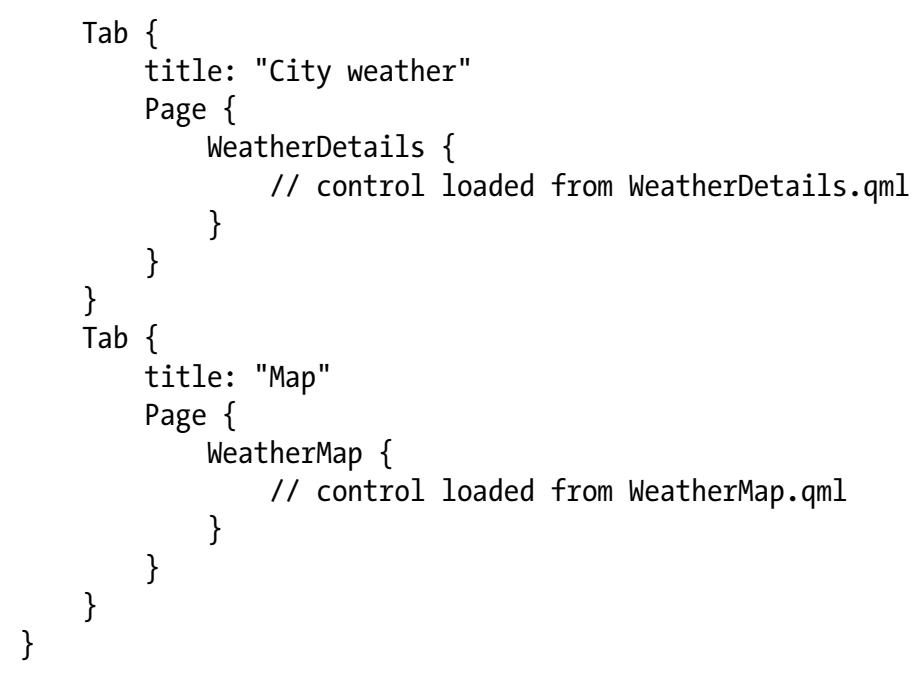

As you can see in Listing 7-9, the WeatherDetails and WeatherMap controls are used as content properties for page controls. The QML engine will therefore automatically load the controls from the corresponding files located in the assets folder of your application project (note that WeatherDetails.qml and WeatherMap.qml are located in the same folder as main.qml).

Let us now have a look at the WeatherDetails control implementation (see Listing 7-10).

\section{Listing 7-10. WeatherDetails.qmI}

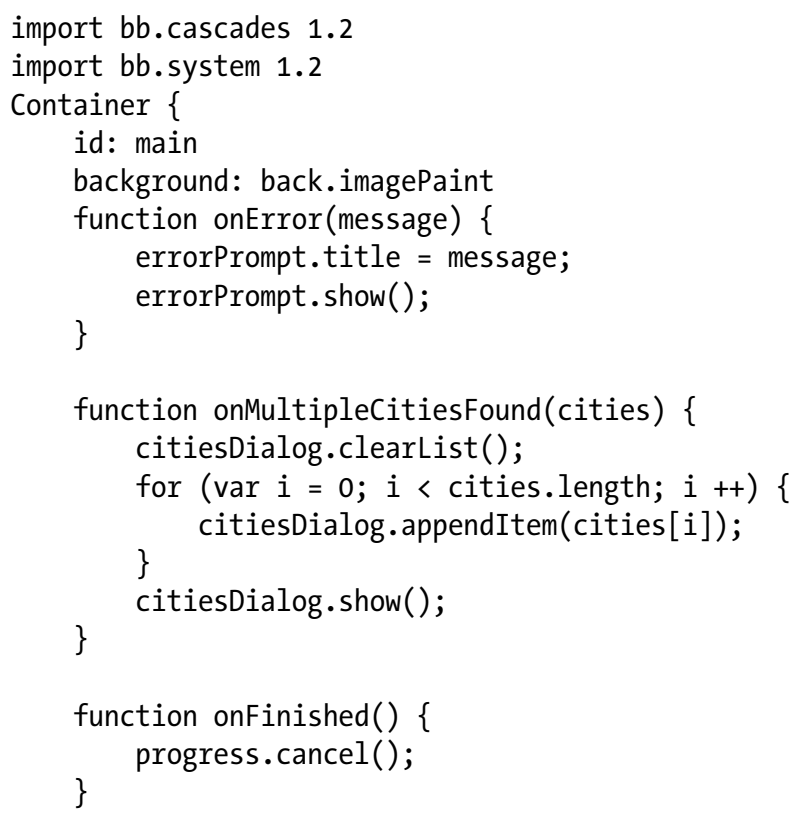




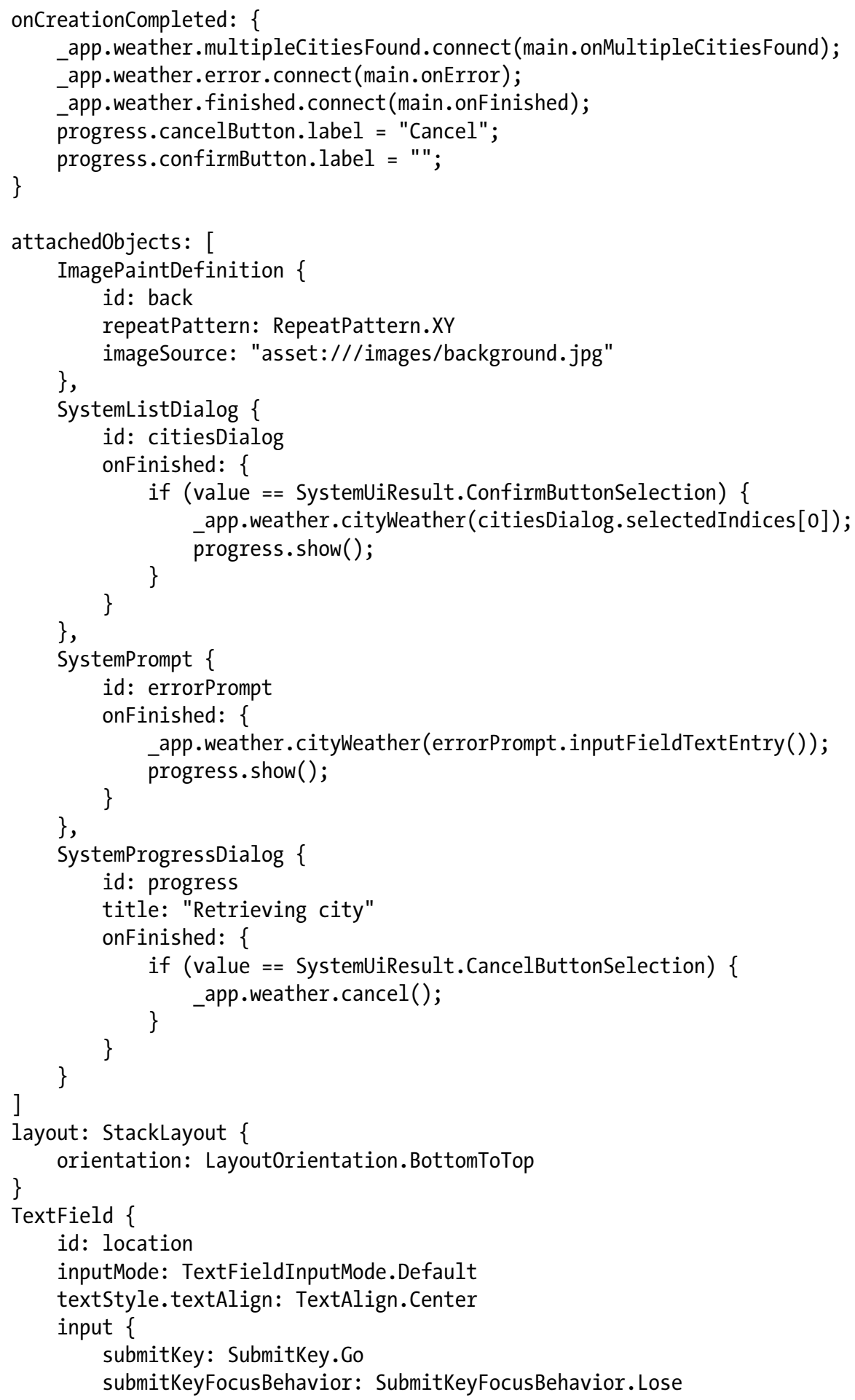




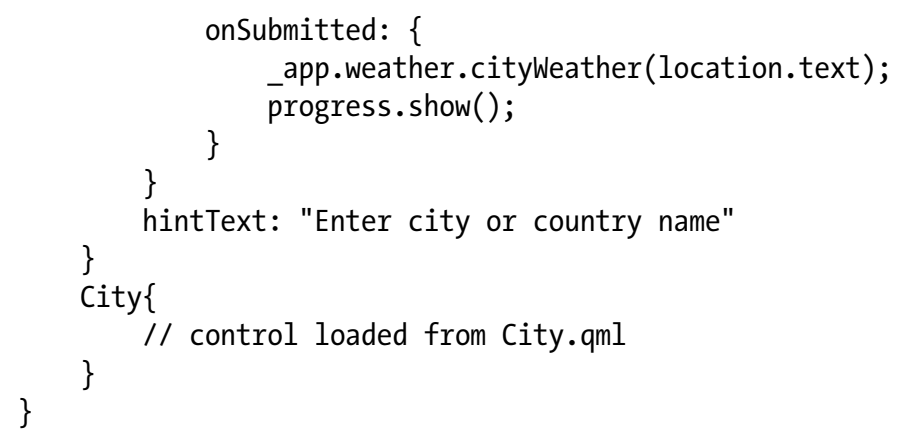

As you can see, WeatherDetails.qml mostly contains some JavaScript code responsible for signal handling. Also, an important point to consider is the way the emitted signals from $\mathrm{C}++$ are connected to the JavaScript functions in the main container's onCreationCompleted slot (in other words, the onError(), onMultipleCitiesFound(), and onFinished() JavaScript functions or slots for signals emitted by the app.weather $\mathrm{C}++_{+}$object). Also note how the location text field's onSubmitted slot is used for calling the app.weather.cityweather() slot, which is defined in C++. If the user's initial query returns multiple cities, a SystemListDialog is displayed, asking him to further refine the query. In the same manner, if an error occurs because the user's query is incorrect, a SystemPrompt is displayed, asking him to correct the query. In both cases,_app.weather.cityweather $($ () is called with the user's updated query.

The City control is mostly a visual control for displaying the results of a weather request: the control uses labels and an image view for displaying the weather conditions for a given city. All QML properties defined in the control are bound to corresponding $\mathrm{C}++_{+}$properties (for example, Listing 7-11 gives you the binding for the current temperature).

\section{Listing 7-11. City Control, Binding Example}

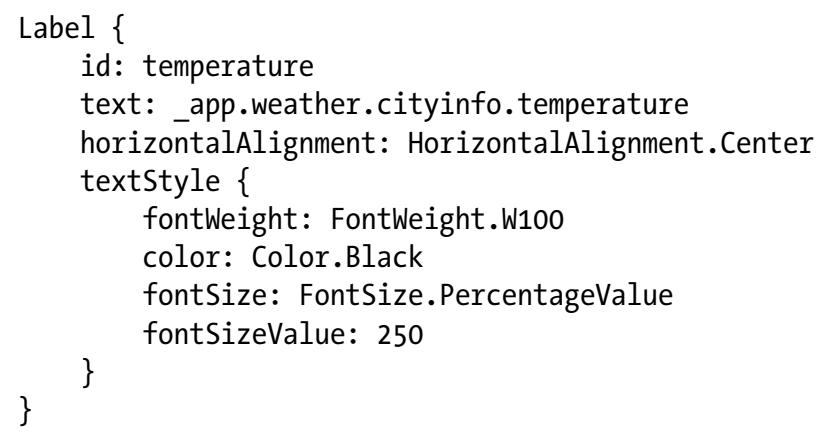

In the example provided in Listing 7-11, the label's text property is bound to the app.weather.cityinfo.temperature property, which is defined in $\mathrm{C}++$ (as you will see in a moment). Therefore, when the _app.weather.cityinfo.temperature property is updated in $\mathrm{C}++$, the QML declarative engine automatically updates the label's text property.

The final QML component to consider is the WeatherMap component, which appears on the second tab. Listing 7-12 gives you component definition. 


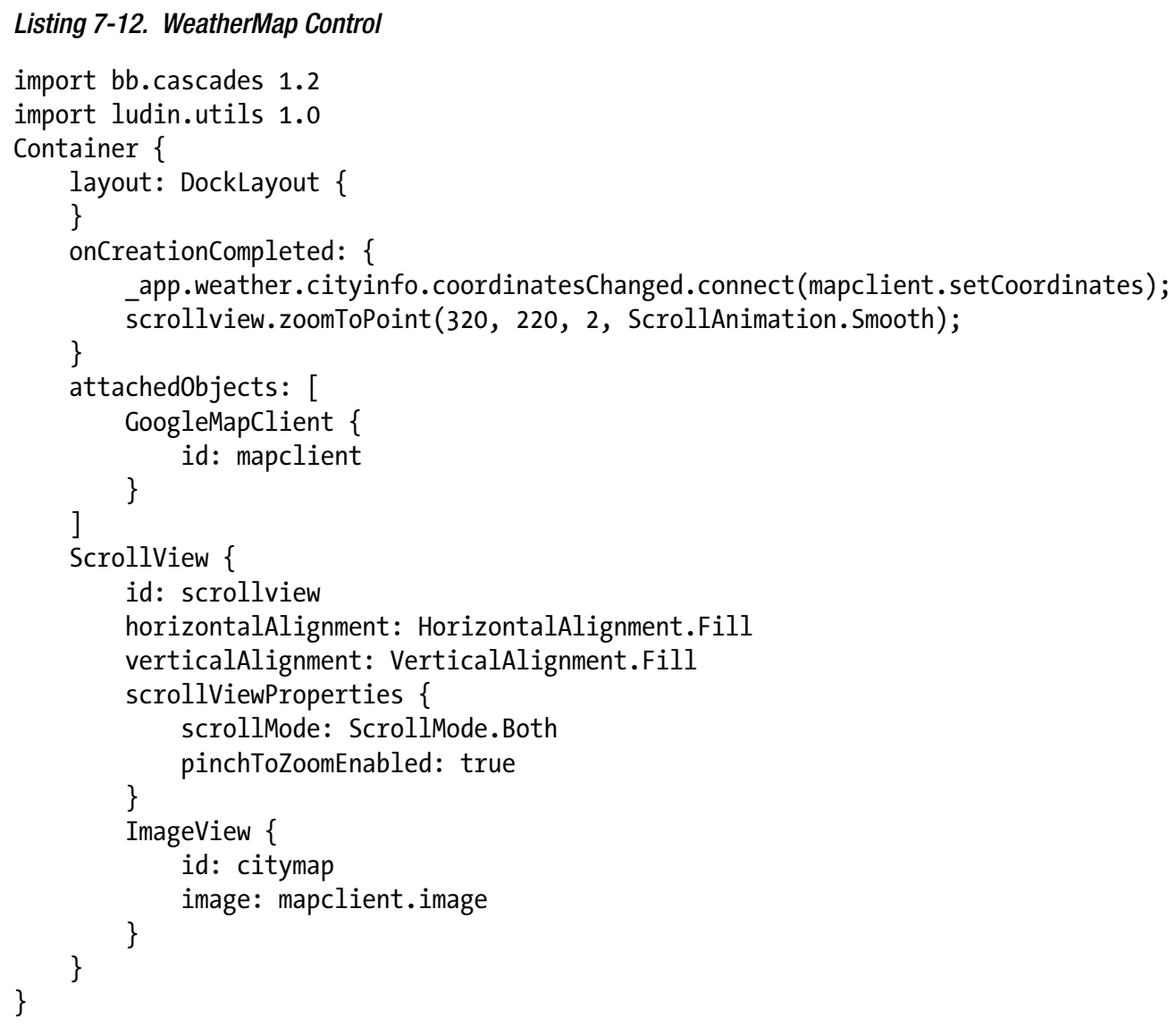

Here again, the control is relatively simple. It mainly consists of an image view responsible for displaying a map of the current weather conditions for a given location. The GoogleMapClient attached object provides the actual weather image. Once again, QML bindings are used to synch the image view and the image map generated by the GoogleMapClient attached object. Finally, the current map coordinates are provided to the GoogleMapClient attached object by the app.weather.cityinfo.coordinatesChanged() signal (the signal to the slot connection is done in the main container's onCreationCompleted slot).

\section{Adding the C++ Implementation}

Let us now turn our attention to the $\mathrm{C}++$ implementation. The most important factor to consider is how to organize your code so that you can define classes with specific responsibilities:

WeatherClient: Responsible for performing the REST requests to the Weather Underground service (www. wunderground.com/weather/api). The class also handles the parsing of the JSON response.

- CityInfo: Encapsulates the weather data once it has been returned by the Weather Underground service. Note that the QML City control has its properties bound to CityInfo's properties. 
GoogleMapClient: A client for generating static maps using the Google Maps service. An instance of this class is defined as an attached object property of the WeatherMap control.

- ApplicationUI: The standard application delegate reachable from the QML layer of your application through the QML document context.

The class relationships are also quite simple: the ApplicationUI object has a WeatherClient weather property, which in turn has a CityInfo property. The properties are accessible from QML as _app.weather and_app.weather.cityinfo, respectively.

\section{WeatherClient}

The WeatherClient class definition is given in Listing 7-13.

\section{Listing 7-13. WeatherClient Class Definition}

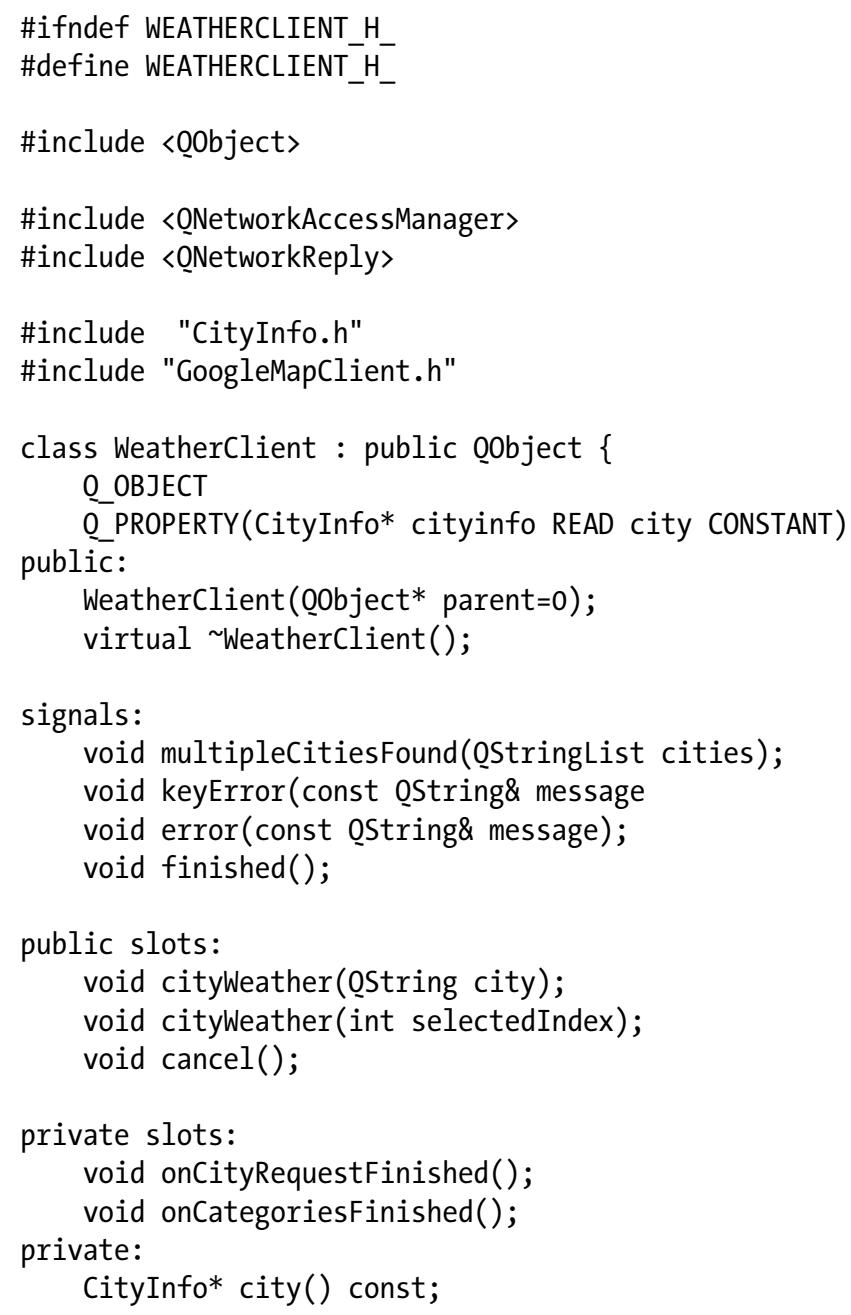




\begin{abstract}
void updateCityInfo(const QVariantMap\& map);
OString m_apiKey;

ONetworkAccessManager* m_networkManager;

OList<ONetworkReply*> m_networkReplies;

CityInfo* m_cityInfo;

OStringList m_cities;

// static char* constant tags omitted

\};

\#endif /* WEATHERCLIENT_H_ */

The class definition declares multiple slots and signals. To perform an initial weather request, the WeatherClient: : cityweather(OString city) slot has to be called from QML (you might recall from Chapter 3 that $\mathrm{C}++$ slots and functions marked as 0 INVOKABLE can be called from QML). Also note that the signals are the same as those handled in JavaScript by the WeatherDetails control (see Listing 7-10). The multipleCitiesFound signal is emitted when a user query corresponds to multiple cities. (The cities are stored in a OStringList and passed as a parameter to the signal. As soon as the user selects a specific city, the WeatherClient: : cityweather (int selectedIndex) slot is called from QML and a new request is sent to the weather service.) The error signal is emitted when the Weather Underground service returns an error (the error is passed as a OString parameter to the signal), and, finally, the finished signal is emitted when a network request has completed.
\end{abstract}

Let us now turn our attention to the WeatherClient member function definitions.

\title{
Constructor
}

Listing 7-14 gives you the WeatherClient constructor.

\section{Listing 7-14. WeatherClient Constructor}

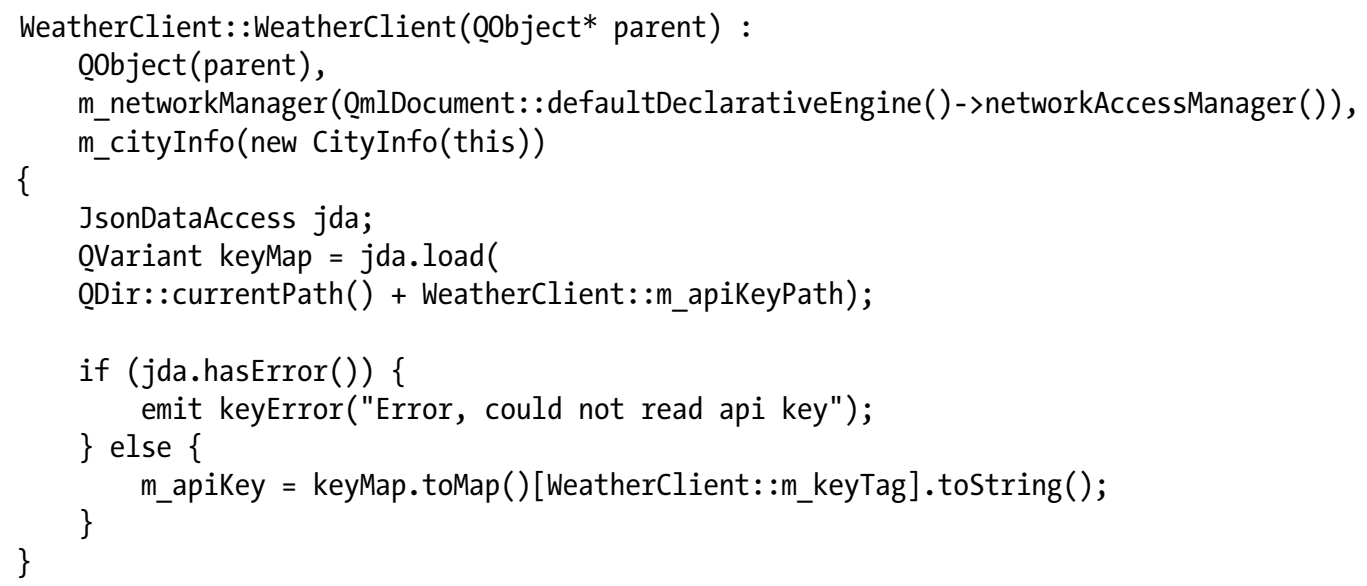


The constructor proceeds by initializing the class members using a member initialization list. The constructor body then tries to load the Weather Underground API key, which is required for each service request. The API key is stored in a JSON file located in a subfolder of your application's assets folder. If the constructor fails to load the key, a signal is emitted so that the UI layer can display an error message to the user. WeatherClient::m_apiKeyPath and Weather: :m_keyTag are string constants that respectively identify the full path to the key file and the corresponding JSON tag.

Note You will need an API key for the Weather Underground service. You will therefore have to create a developer account at www. wunderground.com/weather/api. You will then be able to generate a new key that you can set in the wunderground.json file located in your project's assets/apikey folder.

\section{REST Service Request}

A service request is handled by the WeatherClient: : cityWeather(QString city) member function (see Listing 7-15).

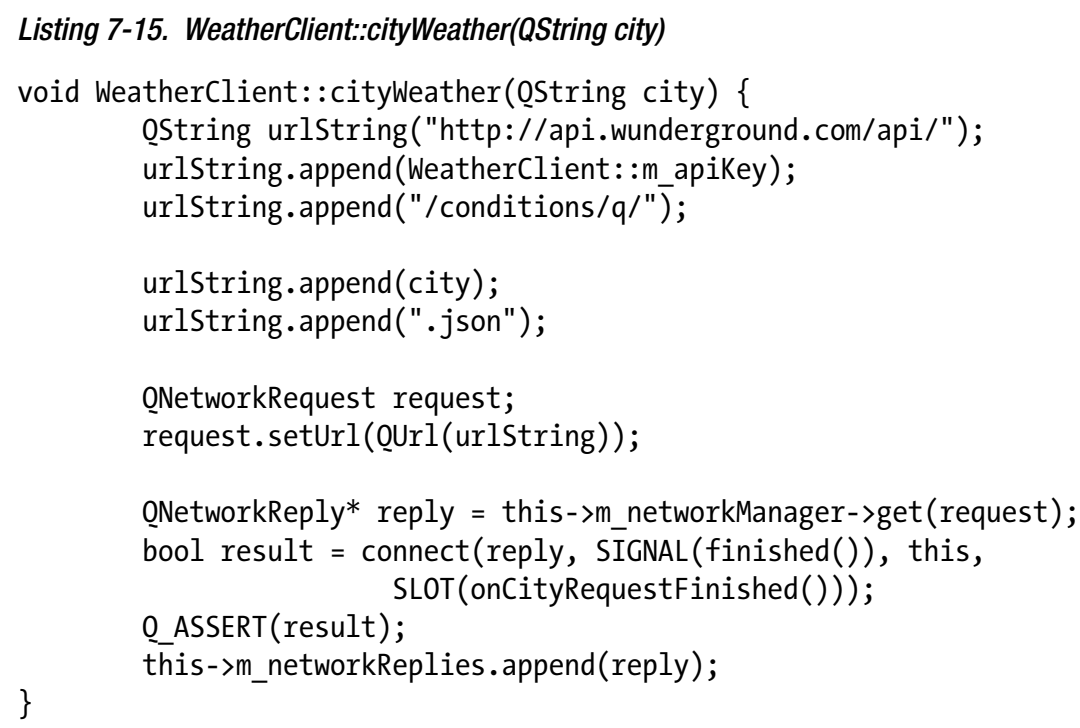

The WeatherClient: : cityweather(OString city) function dynamically creates a GET request URL by concatenating the city parameter and the API key previously loaded in the class constructor (the constructed URL will have the following structure: http://api.wunderground.com/api/ <api key>/conditions/q/<city>.json). As soon as the GET request has been submitted, you will have to connect the ONetworkReply's finished() signal to the WeatherClient's onCityRequestFinished() slot. Finally, when the request has completed, WeatherClient: : onCityRequ estFinished() will be called (see Listing 7-16). 


\section{Working with the Returned JSON}

Before actually looking at how the returned JSON document is parsed by the WeatherClient: : onCityRequestFinished() slot, let us quickly study the structure of the document returned by the Weather Underground service. As a matter of fact, you can conveniently use your browser to perform HTTP requests and study the responses returned by the service. For example, you can use the following URL to retrieve the weather conditions for Los Angeles:

http://api.wunderground.com/api/<key_value>/conditions/q/Los Angeles, CA.json.

The corresponding JSON structure is shown in Listing 7-16 (note that in order to save some page space, I have removed the JSON elements that we will not need to parse or use in our code).

Listing 7-16. Wunderground JSON Response, Single City

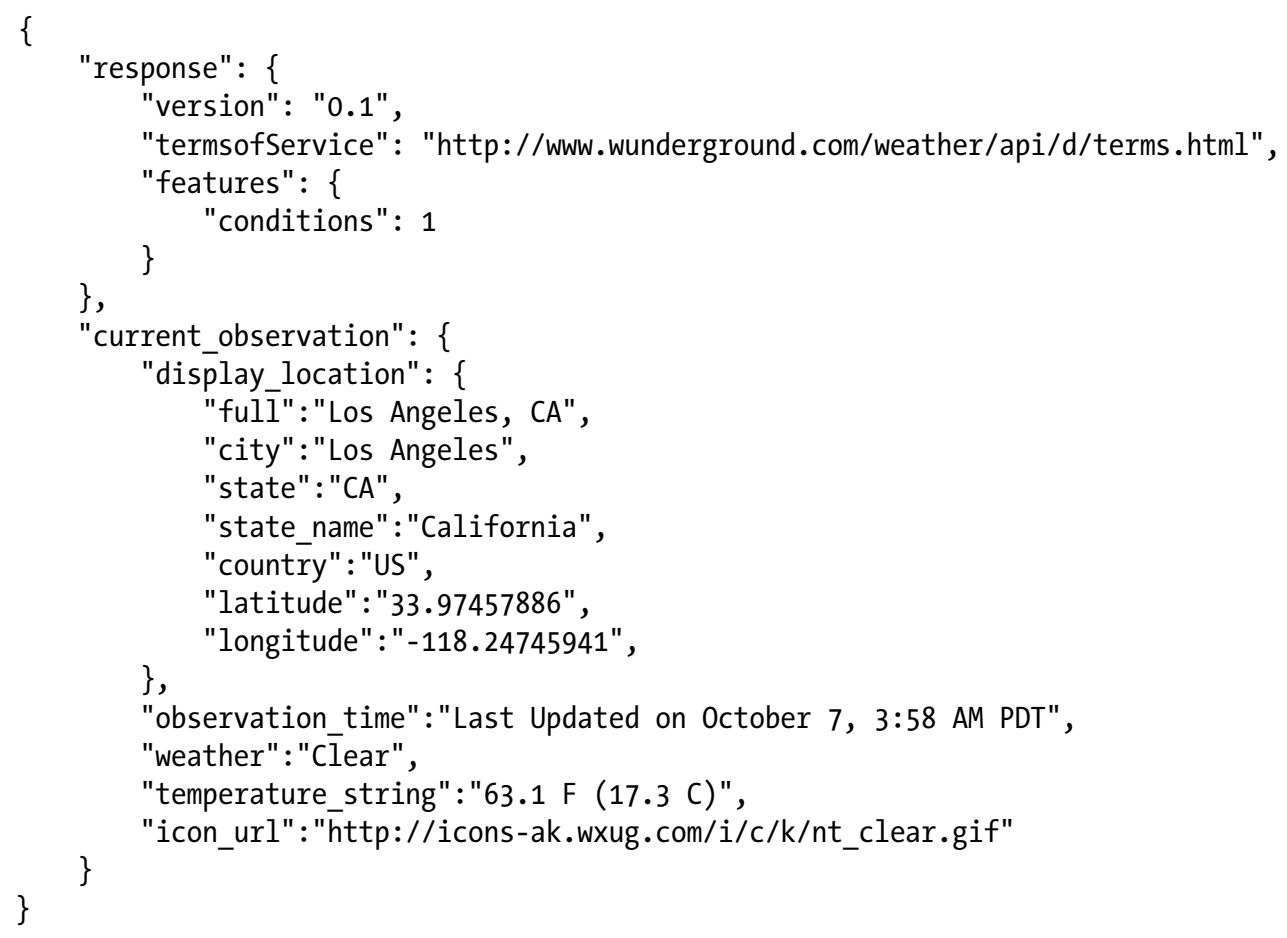

Remembering what I previously told you about parsing JSON documents with a JsonDataAccess object, you can see the following:

From the structure of the document shown in Listing 7-11, the root object is a QVariantMap. Supposing that result is the OVariant variable obtained with the call to JsonDataAccess: : load(), the root object is therefore obtained with a call to result.toMap().

- One level down, the current_observation object contained in the root object is retrieved using result.toMap() ["current_observation"].toMap().

- Similarly, the latitude attribute is retrieved by chaining method calls as follows: result.toMap()["current_observation"].toMap()["display_location"]. toMap()["latitude"].tostring(). 
Once you get the hang of chaining the method calls, you will see that you can parse arbitrarily complex JSON structures.

There will be cases where the JSON response will return a list of cities instead of a single observation (this will happen when the city request matches multiple values). For example, if your request URL is http://api.wunderground.com/api/<key_value>/conditions/q/Los Angeles.json (note the missing state specification), the returned JSON document will be given in Listing 7-17.

\section{Listing 7-17. Wunderground JSON Response, Multiple Results}

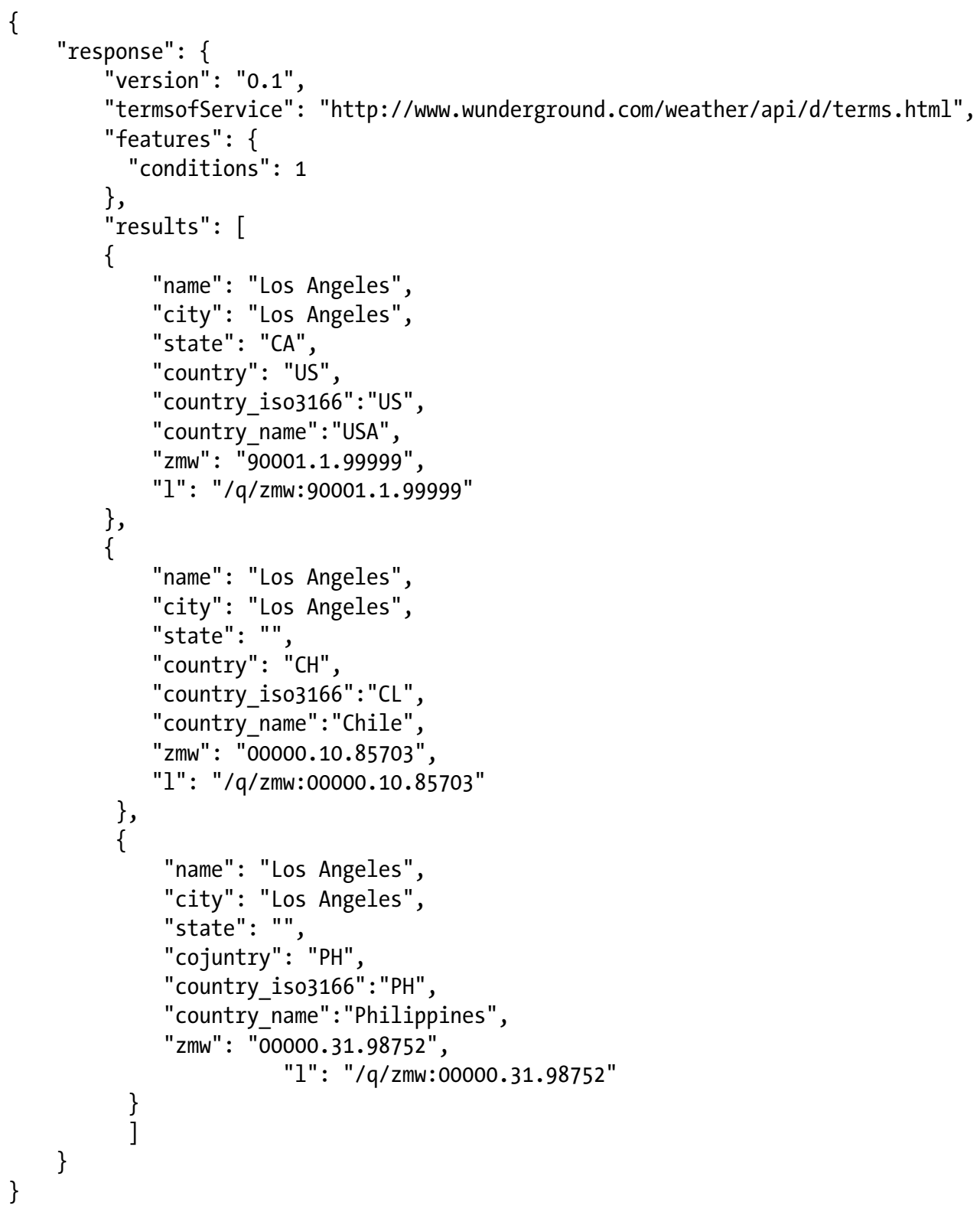


Here again, it is quite easy to retrieve the list of cities using the following call chain:

result.toMap()["response"].toMap()["results"].toList()

And finally, if the request contains an error, the returned JSON document will be similar to Listing 7-18.

Listing 7-18. JSON Response with Error

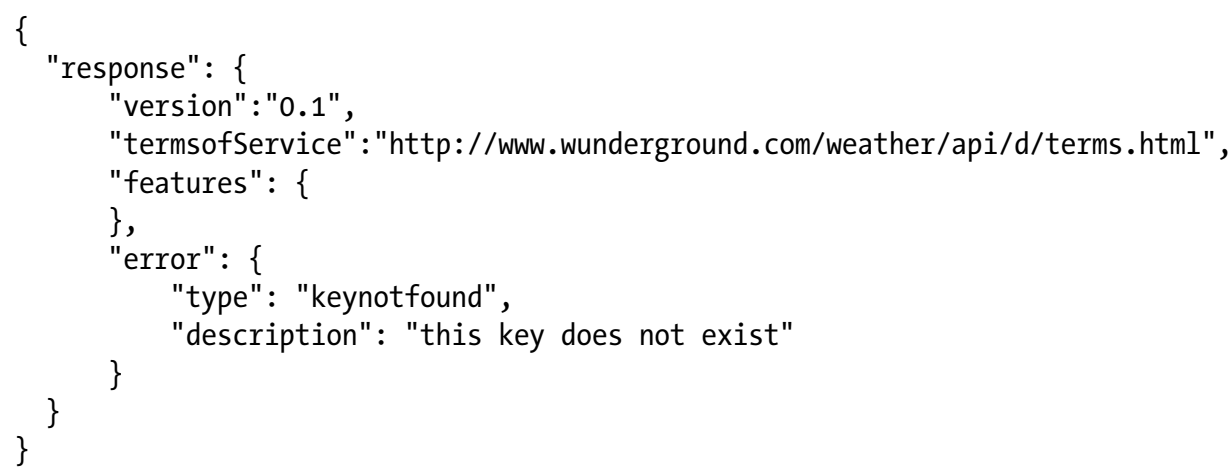

In other words, you can check for the presence of an error object inside the response in order to make sure that your request was handled correctly by the service (the presence of the error object would be given by the following call chain: result.toMap() ["response"].toMap()contains("error")).

Now that you have a basic understanding of the JSON document structure, you can see how the service response is parsed in the WeatherClient: :OnCityRequestFinished() slot (see Listing 7-19).

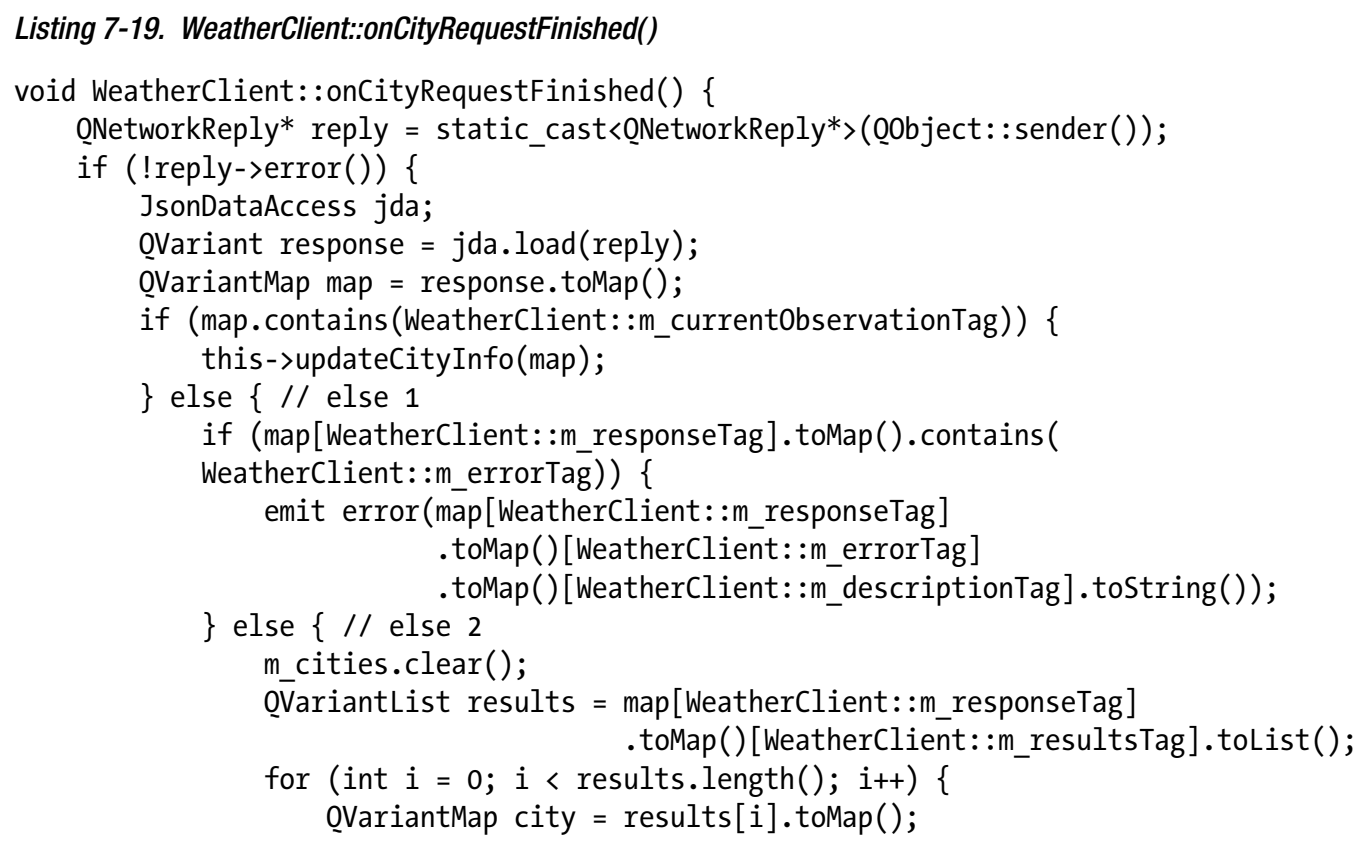




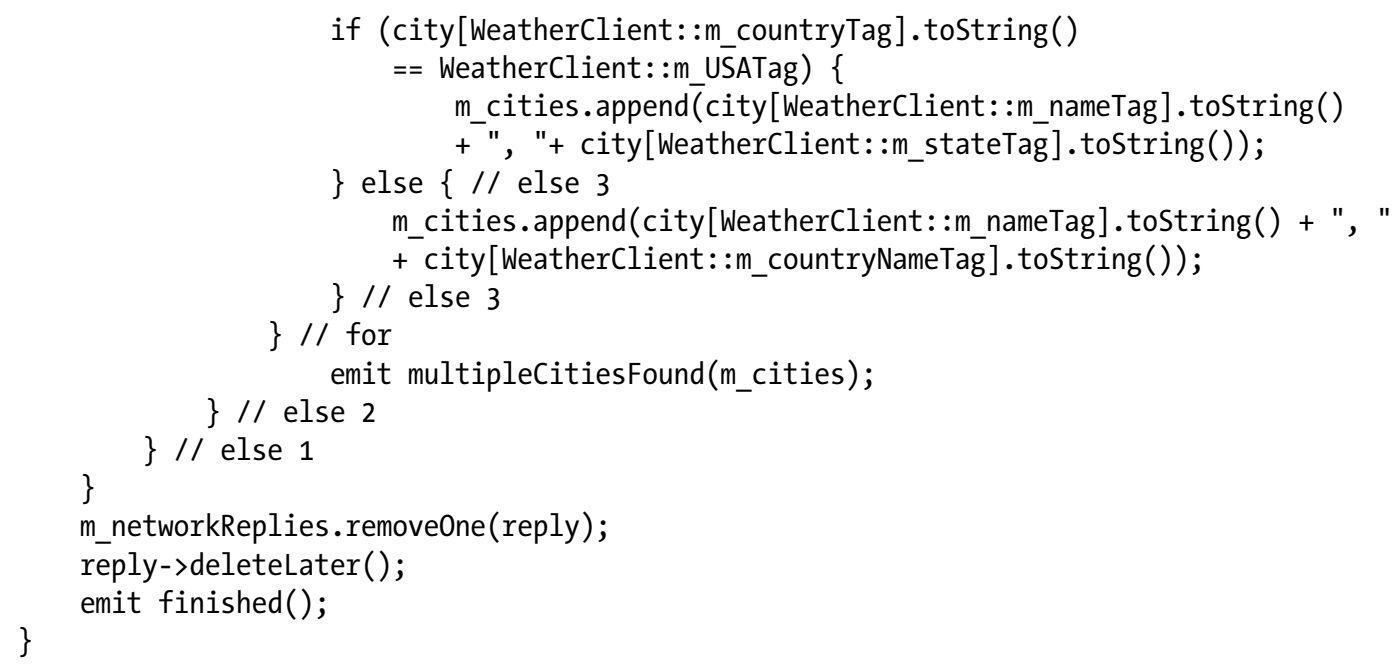

Here is a quick description of the code:

1. You will need to handle three cases in the response: a response can either contain the current weather conditions for a city, a list of cities, or an error object. Before even handling the response, we first need to check that the request was handled correctly and that there are no errors in the ONetworkReply object.

2. We then proceed by parsing the JSON response.

3. If the JSON result contains a current_observation object, we handle it immediately with a call to WeatherClient: : updateCityInfo(const OVariantMap\& map).

4. Otherwise, we check if a service error has occurred. If this is the case, we emit the error signal with the corresponding error message.

5. If there are no errors, then multiples cities have been returned by the request. In this case, we populate the m_citiesList OStringList and emit the multipleCitiesFound(m_citiesList) signal, which will be handled in QML.

6. Finally, we schedule the ONetworkReply object for deletion and emit the finished() signal.

The WeatherService: : updateCityInfo(const QVariantMap\& map) method (used in Listing 7-20) is straightforward and is used for updating the m_cityInfo member variable (which is accessible as the cityinfo property from QML). 


\section{Listing 7-20. WeatherClient::updateCityInfo()}

void WeatherClient: :updateCityInfo(const QVariantMap\& data) \{

OVariantMap currentObservation =

data[WeatherClient::m_currentobservationTag].toMap();

m_cityInfo->setCity (current0bservation[WeatherClient: :m_displayLocationTag] .toMap () [WeatherClient::m_cityTag].toString ());

m_cityInfo->setState(current0bservation[WeatherClient: :m_displayLocationTag] .toMap()[WeatherClient: :m_stateNamēTag].toString( ));

m_cityInfo->setWeather(currentObservation[WeatherClient: :m_weatherTag].toString ( ));

m_cityInfo->setTemperature(current0bservation[WeatherClient: :m_temperatureTag] .tostring ());

m_cityInfo->setCoordinates (currentObservation[WeatherClient: :m_displayLocationTag] .toMap()[WeatherClient: :m_latitudeTag].toString(), currentObservation[WeatherClient::m_displayLocationTag] .toMap ()[WeatherClient: :m_longitudeT̄ag].toString(), currentObservation[WeatherClient: :m_iconUrlTag].toString ()$)$;

m_cityInfo->setLast0bservation(currentObservation[WeatherClient: :m_observationTimeTag] .tostring());

\section{Citylnfo}

Listing 7-21 gives you the CityInfo class definition.

\section{Listing 7-21. CityInfo Class Definition}

\#ifndef CITY_H_

\#define CITY_H_-

\#include $\langle 00 \bar{b} j \overline{e c t}\rangle$

\#include <bb/cascades/Image

\#include 〈QNetworkAccessManager >

class CityInfo : public Qobject \{

O_OBJECT

Q_PROPERTY(OString city READ city NOTIFY cityChanged)

Q_PROPERTY(OString state READ state NOTIFY stateChanged)

Q_PROPERTY(OString latitude READ latitude NOTIFY latitudeChanged)

Q-PROPERTY(OString longitude READ longitude NOTIFY longitudeChanged)

Q_PROPERTY (OString weather READ weather NOTIFY weatherChanged)

O_PROPERTY (OVariant weatherIcon READ weatherIcon NOTIFY weatherIconChanged)

Q_PROPERTY(OString temperature READ temperature NOTIFY temperatureChanged)

Q_PROPERTY(QString lastObservation READ lastObservation NOTIFY lastObservationChanged)

public:

CityInfo(oobject* parent $=0)$;

virtual CityInfo(); 
void setCoordinates(const QString\& latitude, const QString\& longitude, const QString\& weatherIconUrl);

// accessors.

void setCity (const QString\& city);

OString city() const;

void setState(const OString\& state);

QString state() const;

void setLatitude(const OString\& latitude);

OString latitude() const;

void setLongitude(const QString\& longitude);

QString longitude() const;

void setweather(const QString\& weather);

OString weather() const;

void setTemperature(const QString\& temperature);

QString temperature() const;

void setLastObservation(const OString\& lastUpdated);

OString lastObservation() const;

signals:

void cityChanged();

void stateChanged();

void latitudeChanged();

void longitudeChanged();

void coordinatesChanged(const OString\& latitude, const OString\& longitude,

void weatherChanged();

const oString\& markerUrl);

void weatherIconChanged();

void temperatureChanged();

void last0bservationChanged();

private slots:

void onWeatherIconRequestFinished();

private:

QVariant weatherIcon()const;

void setWeatherIconUrl(const OString\& iconUrl);

void downloadWeatherIcon(const QString\& iconUrl);

ONetworkAccessManager* m_networkManager;

OString m_city;

OString m_state;

OString m_latitude;

OString m_longitude;

QString m_temperature; 
QString m_lastObservation;

OString m_weather;

OString m_weatherIconUrl;

bb::cascades::Image m_weatherIcon;

\};

Note that the properties declared in the class definition are the ones used by the QML City control bindings (see Listing 7-11). Also, the Notify signals are required for updating the QML bindings when the $\mathrm{C}++$ properties change.

If you look at Figure 7-3, you will notice that a small icon is used for representing the current weather conditions. The Weather Underground service provides a URL pointing to a downloadable image representing the current conditions (see the icon_url element in the JSON response in Listing 7-16). The Citylnfo class therefore uses the URL to download the icon and display it in QML as an ImageView. Listings 7-22 and 7-23 provide the code for downloading the image.

\section{Listing 7-22. Citylnfo::downloadWeatherlcon}

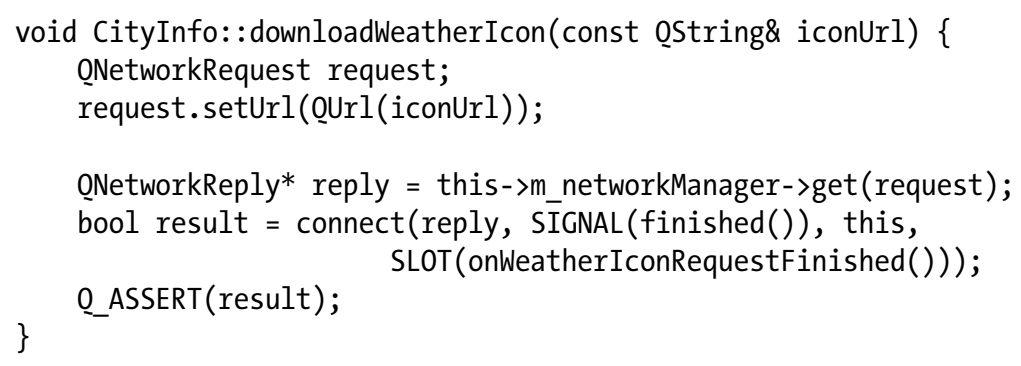

You should be quite familiar by now with the code shown in Listing 7-22. An HTTP request for downloading the image is created and submitted to the network access manager. The interesting part of the code is located in Listing 7-23, which handles the HTTP response.

\section{Listing 7-23. CityInfo::onWeatherlconRequestFinished}

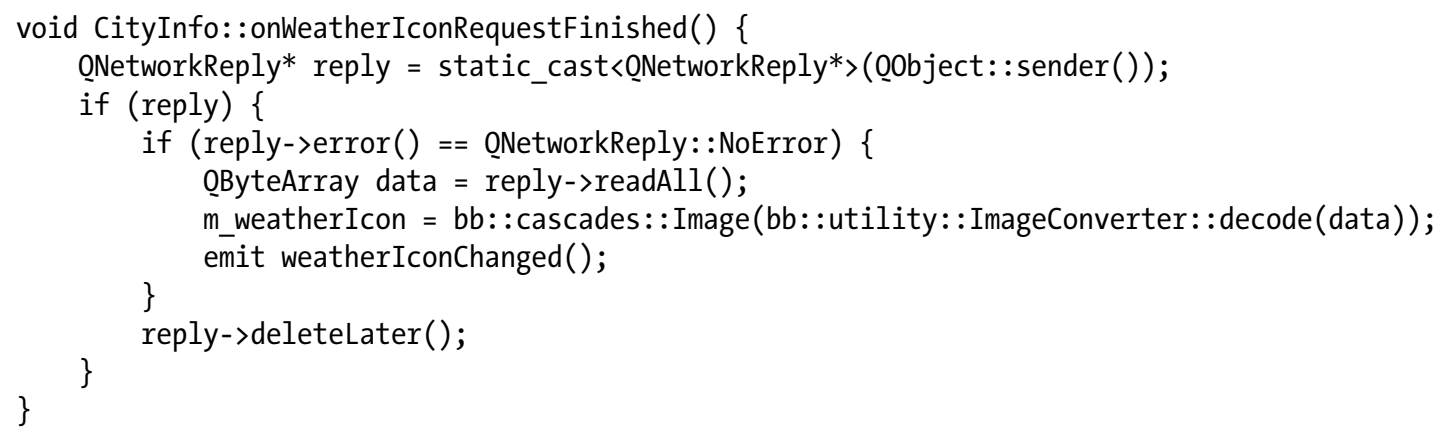

The code essentially builds a bb: :cascades: : Image from the returned data using a bb: : utility: : ImageConverter class, and updates the m_weatherIcon member variable. Note that we also need to emit the weatherIconchanged signal, which will in turn notify the declarative engine to update the QML binding for the City. weather Image property. 
The last piece of the puzzle is to access the Image object as a QVariant from QML using the weatherlcon property (see Listing 7-24).

\section{Listing 7-24. Citylnfo::onWeatherlcon()}

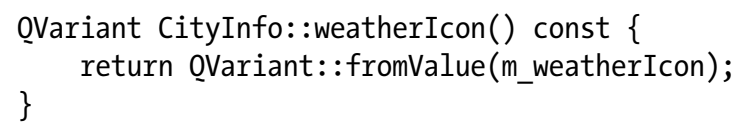

\section{GoogleMapClient}

The GoogleMapClient class generates a static map using the coordinates returned by the Weather Underground service. Here again, the class encapsulates the map generation functionality and exclusively uses properties and signals to communicate with the QML layer. When the GoogleMapClient: : setCoordinates () slot is called, a new request to the Google Maps service is sent. If you look at the WeatherMap control's onCreationCompleted slot, you will notice that the CityInfo: : coordinatesChanged() signal is connected to the GoogleMapClient: : setCoordinates() slot (see Listing 7-12) (in other words, the GoogleMapClient(): : setCoordinates() slot will be called each time the CityInfo object's coordinates are updated).

Listing 7-25 shows you the GoogleMapClient: : setCoordinates() slot implementation.

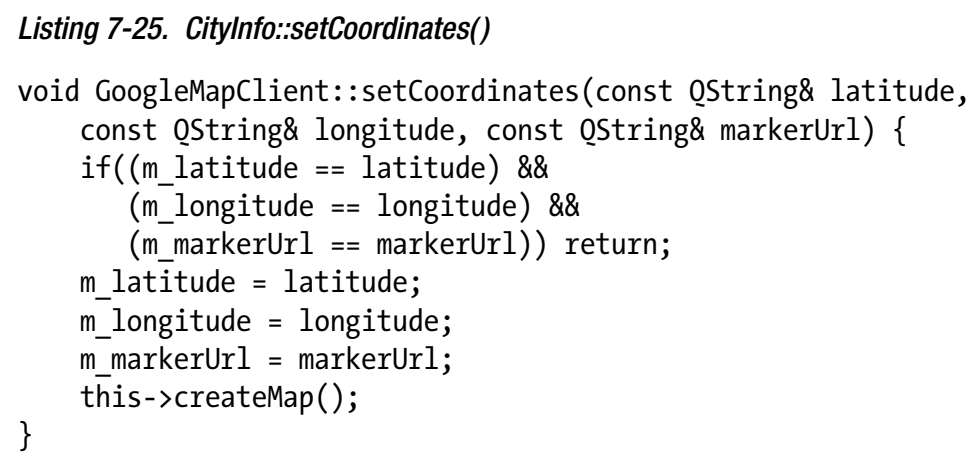

Finally, the GoogleMapClient: :setCoordinates() method internally calls the GoogleMapClient: :createMap() method, which is responsible for building the network request to the Google Maps service (see Listing 7-26).

\section{Listing 7-26. GoogleMapClient::createMap()}

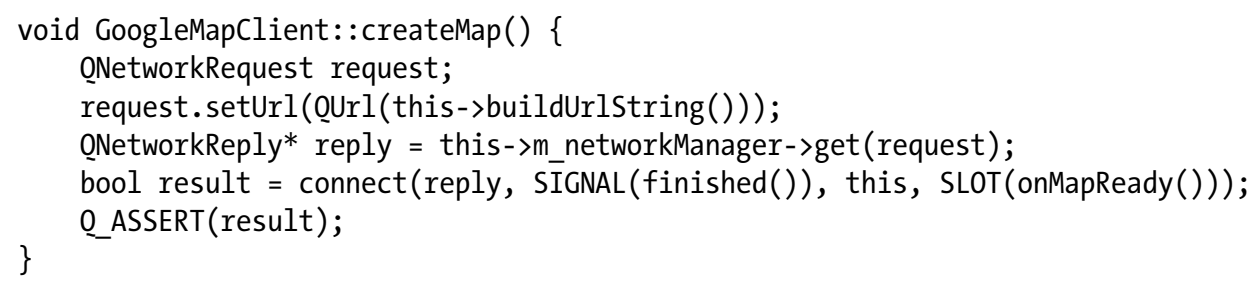


I am going to omit the code for handling the HTTP response, which is done in GoogleMapClient: : onMapReady (), because it is very similar to WeatherClient: : onWeatherIconReque sFinished() (shown in Listing 7-23). (In retrospect, we could have designed a common base class implementing the image download logic. This is something you could try to refactor.)

The request URL is built with a call to GoogleMapClient: : buildUrlString() (see Listing 7-27).

\section{Listing 7-27. GoogleMapClient::buildUrlString()}

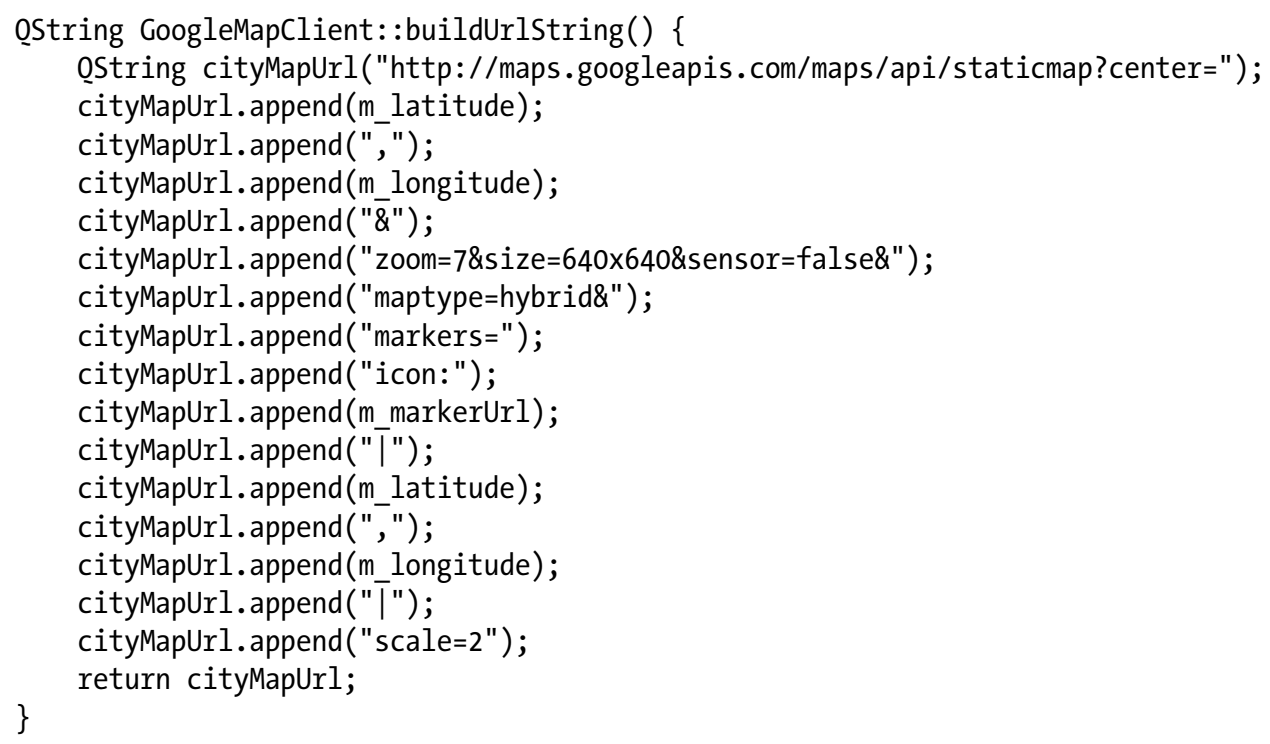

The code shown in Listing 7-27 essentially creates a new request for a map centered on the

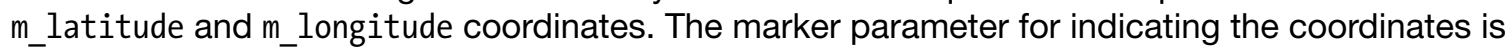
defined as the URL of the icon returned by the Weather Underground service. (If you specify an image URL as a marker, Google Maps will add it as a marker on your map. By default, when no markers are specified, Google will use its own for the coordinates). This illustrates how you can combine, in practice, multiple services in your own app (we could say that we have built a mashable app).

If you are interested in finding out more about the Google static maps API, you can refer to the following URL: https://developers.google.com/maps/documentation/staticmaps.

\section{ApplicationUI}

As usual for Cascades applications, the application delegate ties everything together and provides you the access point for the WeatherClient and CityInfo instances (note that the delegate itself is set as a QML document context property; see Listings 7-28 and 7-29).

\section{Listing 7-28. ApplicationUl Definition}

class ApplicationUI : public QObject

\{

Q_OBJECT

Q_PROPERTY (WeatherClient* weather READ weatherClient CONSTANT) 


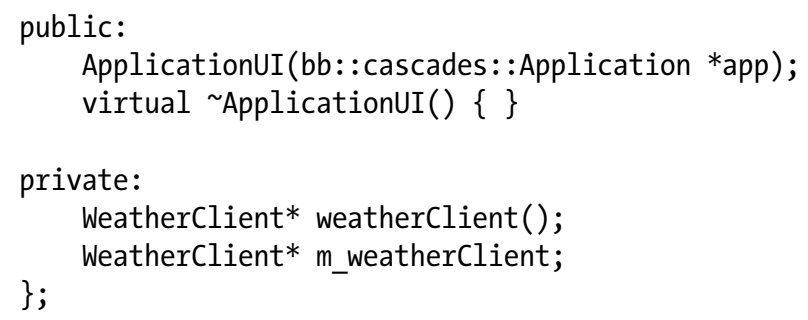

\section{Listing 7-29. ApplicationUI Constructor}

ApplicationUI::ApplicationUI(bb::cascades: :Application *app) :

oobject(app), m_weatherClient(new WeatherClient(this)) \{

// Create scene document from main.gml asset, the parent is set

// to ensure the document gets destroyed properly at shut down.

OmlDocument $*$ gml = QmlDocument: :create("asset:///main.qml").parent(this);

qml->documentContext()->setContextProperty("_app", this);

// Create root object for the UI

AbstractPane ${ }^{*}$ root $=$ qml- $>$ createRootObject $\langle$ AbstractPane $\rangle()$;

// Set created root object as the application scene

\} app->setScene(root);

Finally, to make the WeatherClient, CityInfo and GoogleMapClient classes available as new QML types, you need to register them with the QML type system. This is done in the application's main function (see Listing 7-30).

\section{Listing 7-30. main.cpp}

O_DECL_EXPORT int main(int argc, char **argv)

qmlRegisterType<CityInfo>("ludin.utils", 1, 0, "CityInfo");

qmlRegisterType<WeatherClient>("ludin.utils", 1, 0, "WeatherClient");

qmlRegisterType<GoogleMapClient>("ludin.utils", 1, 0, "GoogleMapClient");

Application app(argc, argv);

// Create the Application UI object, this is where the main.qml file

$/ /$ is loaded and the application scene is set.

new ApplicationUI(\&app);

// Enter the application main event loop.

\} return Application: $\operatorname{exec}()$; 
The first two calls to qmlRegisterType() are required because you are using CityInfo and WeatherClient as properties accessible from QML. The last call is required so that you can define the GoogleMapClient class as an attached object in the WeatherMap control. (You also need to add the import ludin.utils 1.0 statement at the start of your QML document; see the WeatherMap control in Listing 7-12.)

\section{Summary}

This chapter provided an overview of the BlackBerry 10 networking classes based on the QtNetwork module. The networking classes are completely generic, but this chapter showed you how to use them for the HTTP protocol. ONetworkManager plays the role of the grand dispatcher to submit network requests and handle responses. The class supports the usual HTTP verbs (GET, PUT, and POST), which makes it a breeze to use with restful services. An HTTP request is encapsulated by a ONetworkRequest instance and the response can be handled using a corresponding ONetworkReply instance. Networking is completely asynchronous, thus ensuring that UI thread is not blocked during an HTTP request. Finally, it should be emphasized that the networking classes are reentrant, meaning that you can call them multiple times from a single thread without corrupting their state. 